\title{
BMJ Open Effectiveness of problem gambling interventions in a service setting: a protocol for a pragmatic randomised controlled clinical trial
}

\author{
M Abbott, ${ }^{1,2} \mathrm{M}$ Bellringer, ${ }^{1,2} \mathrm{~A} C$ Vandal, ${ }^{2,3,4} \mathrm{DC}$ Hodgins, ${ }^{5} \mathrm{M}$ Battersby, ${ }^{6}$ \\ S N Rodda ${ }^{1,2}$
}

To cite: Abbott $M$ Bellringer M, Vandal AC, et al. Effectiveness of problem gambling interventions in a service setting: a protocol for a pragmatic randomised controlled clinical trial. BMJ Open 2017;7:e013490. doi:10.1136/bmjopen-2016013490

- Prepublication history for this paper is available online. To view these files please visit the journal online (http://dx.doi.org/10.1136/ bmjopen-2016-013490).

Received 15 July 2016 Revised 11 January 2017 Accepted 9 February 2017

\section{CrossMark}

For numbered affiliations see end of article.

Correspondence to Professor M Abbott; max. abbott@aut.ac.nz

\section{ABSTRACT \\ Introduction: The primary purpose of this study is to evaluate the relative effectiveness of 2 of the best developed and most promising forms of therapy for problem gambling, namely face-to-face motivational interviewing (MI) combined with a self-instruction booklet (W) and follow-up telephone booster sessions $(\mathrm{B} ; \mathrm{Ml}+\mathrm{W}+\mathrm{B})$ and face-to-face cognitive-behavioural therapy (CBT).}

Methods and analysis: This project is a single-blind pragmatic randomised clinical trial of 2 interventions, with and without the addition of relapse-prevention text messages. Trial assessments take place pretreatment, at 3 and 12 months. A total of 300 participants will be recruited through a community treatment agency that provides services across New Zealand and randomised to up to 10 face-to-face sessions of CBT or 1 face-toface session of $\mathrm{Ml}+\mathrm{W}$ +up to $5 \mathrm{~B}$. Participants will also be randomised to 9 months of postcare text messaging. Eligibility criteria include a self-perception of having a current gambling problem and a willingness to participate in all components of the study (eg, read workbook). The statistical analysis will use an intent-to-treat approach. Primary outcome measures are days spent gambling and amount of money spent per day gambling in the prior month. Secondary outcome measures include problem gambling severity, gambling urges, gambling cognitions, mood, alcohol, drug use, tobacco, psychological distress, quality of life, health status and direct and indirect costs associated with treatment.

Ethics and dissemination: The research methods to be used in this study have been approved by the Ministry of Health, Health and Disability Ethics Committees (HDEC) 15/CEN/99. The investigators will provide annual reports to the HDEC and report any adverse events to this committee. Amendments will also be submitted to this committee. The results of this trial will be submitted for publication in peerreviewed journals and as a report to the funding body. Additionally, the results will be presented at national and international conferences.

Trial registration number: ACTRN12615000637549.
Strengths and limitations of this study

- As a pragmatic trial, this study can determine the effectiveness of cognitive-behavioural and motivational treatments for problem gambling when delivered in a community setting.

- The proposed sample size will permit subgroup analyses of a range of potential mediators and moderators.

- The study examines the use of text messaging as a method of extending treatment and preventing relapse.

- Recruitment within one large clinical setting may have an impact on speed of participant recruitment.

\section{INTRODUCTION}

Problem gambling and wider gambling-related harms constitute a significant health and social problem in many parts of the world. ${ }^{2}{ }^{2}$ The New Zealand National Gambling Study indicates that disordered gambling contributes to, and is associated with, a broad spectrum of morbidity and harm to individuals, families and communities. ${ }^{3}$ The 2011/2012 New Zealand Health Survey found that problem gamblers are nearly four times more likely to smoke and almost five times more likely to engage in hazardous alcohol consumption than nonproblem gamblers. ${ }^{4}$ People with problem gambling have much higher rates of a number of mental health conditions, particularly substance misuse, affective and anxiety disorders. ${ }^{5}{ }^{6}$ These rates are typically higher in help-seeking settings than in general population studies.

Psychological treatment for problem gambling includes various combinations of cognitive-behavioural therapies (CBTs) and motivational approaches such as motivational 
interviewing (MI). These treatments have moderate-to-high effect size (ES) and are better than no-treatment controls. ${ }^{78}$ A recent study by Battersby and colleagues ${ }^{9}$ combined cognitive restructuring ${ }^{10}{ }^{11}$ and graded exposure with response prevention. This programme is designed to be flexible, benefit from synergies between the behavioural and cognitive elements and enhance treatment adherence.

MI has attracted less research attention than CBT. A Cochrane review identified just four studies involving MI (out of a possible 14) and concluded that MI may have a positive impact on gambling behaviour, but there was insufficient evidence of it having an effect on other gambling impacts (eg, depression or anxiety). ${ }^{8}$ The review focused on studies involving clients presenting to face-to-face services and did not take into account studies involving telephone or online administration. ${ }^{12-14}$ When delivered with other resources or support, such as a behavioural and cognitive strategies workbook or additional post-treatment support sessions, MI results in a significant reduction in gambling behaviour that is sustained over a longer time period. ${ }^{15}$ A recent telephone trial reported that MI plus a workbook plus booster telephone sessions $(\mathrm{MI}+\mathrm{W}+\mathrm{B})$ resulted in significantly better outcomes for Māori (New Zealand's indigenous population), people with more severe gambling problems, higher comorbidity and those who sought to moderate rather than stop gambling. ${ }^{16}$

There are few efficacy, effectiveness or outcome studies that have examined the longer term durability of treatment effects. Cochrane and other reviews have noted that these interventions are 'possibly efficacious' because no intervention has been demonstrated to meet formal efficacy or effectiveness standards. In addition, there are very few studies that have evaluated longer term outcomes or developed or assessed ways to sustain treatment effects and prevent relapse. A number of studies have failed to demonstrate outcome differences between different types and intensities of treatment for problem gambling. ${ }^{16-19}$ How much of this is a consequence of a lack of statistical power and other design deficiencies rather than lack of difference in therapeutic potency is unclear.

Prospective general population studies indicate that for most people problem gambling has a fluctuating natural history, with many people transitioning into and out of problem states of varying severity and duration. ${ }^{320}$ While natural recovery rates appear to be high, greater problem severity and comorbidity are associated with chronicity and relapse. ${ }^{21}$ This highlights the importance of developing interventions that maintain treatment outcomes and reduce the frequency of relapse. Text messaging has been posited as an easy method of extending treatments and reducing relapse and has been associated with positive outcomes in tobacco, but less so for a study involving a small sample size and alcohol misuse. ${ }^{22-25}$ A recent study offered 12 change strategies over a 12-week period to gamblers accessing online counselling (ie, a single session of chat, email, community forums or very brief self-help). ${ }^{26}$ This study reported that text messages did not lead to greater improvement in gambling symptoms or behaviour over and above the online treatment accessed. These findings were possibly due to a small sample size, frequency of messages and lack of message tailoring (ie, to their readiness to change).

There is an urgent need to determine the most effective treatments for gambling disorder and also determine how they can be widely adopted in practice settings. The current study is designed to address the shortcomings in the research base underlying treatment for problem gambling. Shortcomings include a small sample size leading to low statistical power, heterogeneous samples, high attrition, inadequate follow-up, lack of protocol driven treatments, missing or skewed data, single site clinical trials, failure to include comparative or control groups and intention-to-treat (ITT) analyses, lack of study replication by independent investigators and high rates of non-specific treatment response. It will extend research by taking promising interventions that have been examined in efficacy studies with predominantly non-clinical volunteers and evaluating them in clinical settings. Moreover, the study will determine effectiveness and involve community-based counsellors and clients seeking help from gambling counselling services.

The primary aim of this trial is to evaluate the relative effectiveness of two of the best developed and most promising forms of therapy for problem gambling, namely face-to-face $\mathrm{MI}+\mathrm{W}+\mathrm{B}$ and face-to-face CBT. Secondary aims of this study are to evaluate the effectiveness of gambling-related post-treatment text messaging in preventing relapse and sustaining treatment gains at 12 months and evaluate the extent to which common comorbidities diminish following therapy. In addition, engagement will be the potential moderator and mediator. The study will also identify which, if any, of the interventions is more effective for a variety of client ethnic groups including Māori and Pacific, and groups identified by problem severity and comorbidities. Additionally, it will identify the relationship between changes in potential mediators of treatment response including comorbidities and reductions in gambling participation and problems. The primary hypothesis is that CBT participants will show greater clinically meaningful reductions in gambling and problem gambling than $\mathrm{MI}+\mathrm{W}+\mathrm{B}$ participants at 12 months. Secondary hypotheses are:

- $\mathrm{CBT}$ and $\mathrm{MI}+\mathrm{W}+\mathrm{B}$ will be equivalent with respect to reductions in gambling and problem gambling at 3 months;

- Participants allocated to the post-treatment text messaging conditions, in the CBT and $\mathrm{MI}+\mathrm{W}+\mathrm{B}$ groups, will show greater clinically meaningful reductions in gambling and problem gambling at 12 months than those in the non-text messaging condition;

- CBT participants will have greater reductions in depression and anxiety than $\mathrm{MI}+\mathrm{W}+\mathrm{B}$ participants at 12 months. 


\section{METHODS AND ANALYSIS}

\section{Trial design}

We will conduct a single-blind pragmatic randomised clinical trial (RCT) of two interventions, with and without the addition of text messages, and with a prospective follow-up. Trial assessments take place pretreatment, at 3 and 12 months. Therapy interventions, namely face-to-face CBT and a single face-to-face $\mathrm{MI}+\mathrm{W}+5 \mathrm{~B}$, will be conducted over 3 months. Following the 3-month assessments, participants receiving $\mathrm{CBT}$ and $\mathrm{MI}+\mathrm{W}+\mathrm{B}$ who are allocated to the text conditions will receive text messaging for 9 months. Primary outcome measures will be days gambled (self-reported number of days gambled in the previous month) and money spent (self-reported money spent per day in the previous month). These outcomes will be treated as a multivariate outcome in the analysis. The research methods to be used in this study have been approved by the Ministry of Health, Health and Disability Ethics Committees (HDEC) 15/CEN/99.

\section{Participants and recruitment}

Participants will be recruited from people seeking help from the Salvation Army Addiction Services-Gambling (Oasis) for problems with their own gambling. Oasis is a national problem gambling service provider operated by the Salvation Army that provides services to all major ethnicities in New Zealand. Gamblers who contact Oasis centres for treatment will be informed of the trial by the administrator or counsellor with whom they have made initial contact.

Individuals are eligible to participate if they are a minimum age of 18 years; self-perception of having a current gambling problem; willing to read materials related to the study (to ensure reading ability); willing to participate in counselling and other treatment components; willing to have counselling sessions recorded and willing to provide follow-up data on gambling. Participants will also be asked to provide the details of a collateral person (family member or friend) for the purposes of follow-up (staying in touch when contact details change) and as a check on gambling information provided. Participants will not be precluded from taking part in the study if they are not comfortable giving the details of collateral persons. Exclusion criteria will be the presence of active psychosis or active suicidal intent which is routinely assessed at intake at the clinician's discretion. See figure 1 for the study flow chart.

Clients who meet the eligibility criteria and give their verbal consent to participate will be contacted by a trained research assistant who will administer the baseline assessment questionnaire. Screening details required by Oasis and the treatment allocation information will be transferred to Oasis ahead of the client's scheduled appointment. Assessment interviews will be conducted by a research assistant within 7 days from the client's initial contact with the service.
Figure 1 Participant flow for study. CBT, cognitive-behavioural therapy; MI, motivational interviewing.

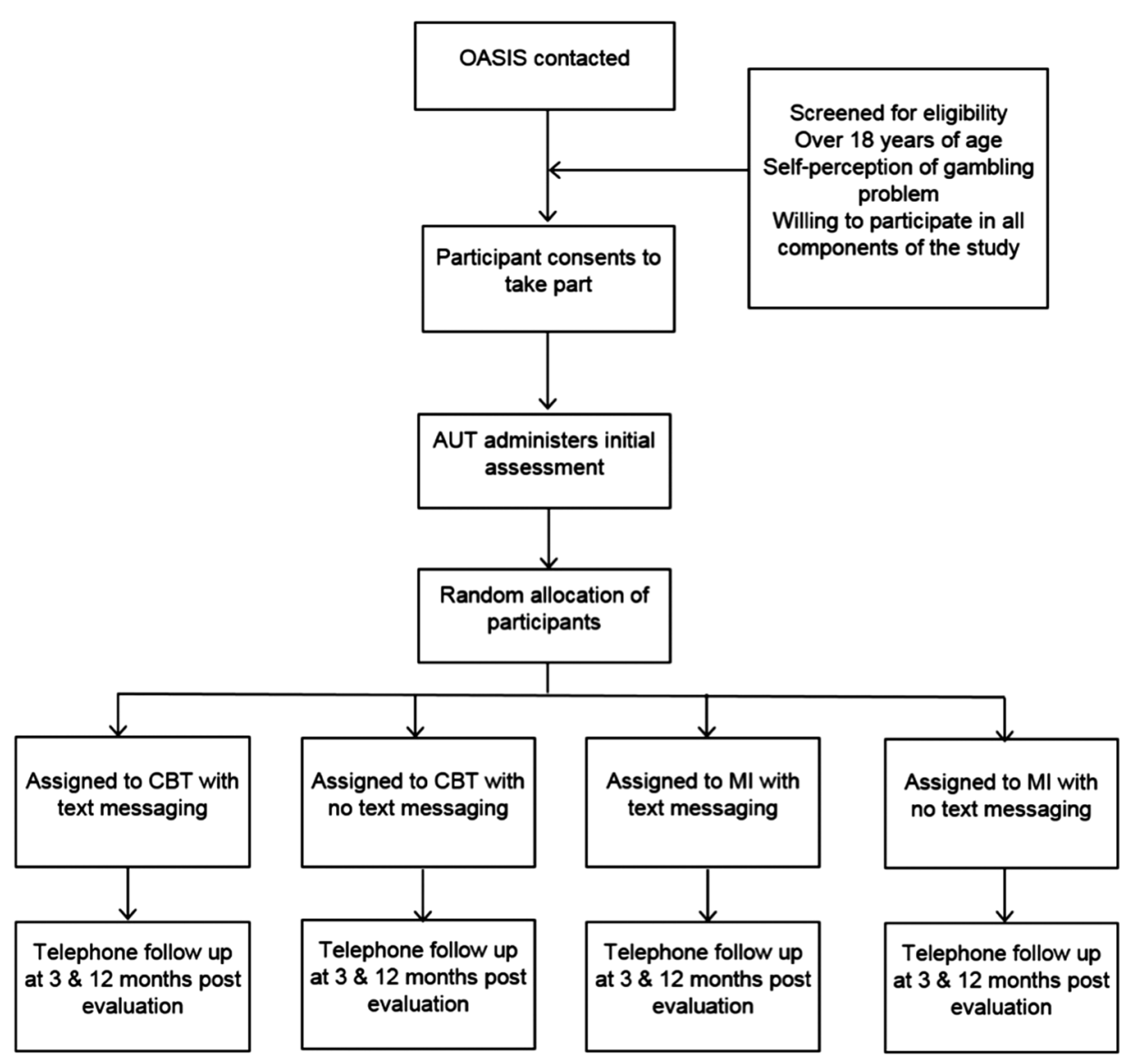




\section{Randomisation}

To ensure equal numbers across the four conditions, block randomisation will be used, with random block sizes varying between 4 and 12 to promote concealment. The block size distribution will be kept secret until the unblinding. Block randomisation will be stratified on recruitment site and self-declared ethnicity defined on three levels: Māori, Pacific and others. The randomisation schedule algorithm will be coded by information technology staff (supervised by the trial statistician) and implemented by an independent party who will select a seed for the pseudorandom number generator and generate the actual schedule. Participants will be allocated to one of the four treatment combinations in a 1:1:1:1 ratio (CBT or $\mathrm{MI}+\mathrm{W}+\mathrm{B}$ plus text messaging, $\mathrm{CBT}$ or $\mathrm{MI}$ $+\mathrm{W}+\mathrm{B}$ without text messaging).

\section{Intervention conditions}

\section{Motivational interviewing plus self-help workbook and}

telephone follow-up

$\mathrm{MI}+\mathrm{W}+\mathrm{B}$ will integrate a single face-to-face motivational interview, a self-help workbook and five follow-up motivational telephone sessions. The first of these follow-up sessions may be delivered face to face rather than by telephone if strongly preferred by the participant. MI will be structured to encourage clients to build a commitment to change by emphasising the reasons why change is desirable and has been previously described by Abbott $e t a l .{ }^{16}$ The self-help workbook was evaluated in a previous New Zealand trial involving helpline callers ${ }^{16}$ and this was based on a resource that has been evaluated multiple times. ${ }^{12}{ }^{14}$ The telephone sessions will focus on motivation of, and reinforcement for, behaviour change through the use of the workbook. They will be offered for 10-50 min duration at $\sim 1,2,4$, 8 and 12 weeks.

\section{Cognitive-behavioural therapy}

The CBT intervention will be up to 10 face-to-face sessions offered for up to $90 \mathrm{~min}$ each session over a 12-week period. Therapy is based on a manual that Battersby and colleagues have developed drawing on behavioural therapy and cognitive therapy. Though the protocol is sequential, the manual allows therapists some flexibility with session sequencing, dependent on client response and progress. The intervention is informed by the outcomes of a pilot RCT and previous clinical trials ${ }^{1117}$ and trialled with 41 state-wide gambling service treatment patients in Australia. As described in table 1, it incorporates imaginal and real-life cue exposure to gambling triggers and habituation/urge extinction. It also includes interventions directed towards understanding randomness and erroneous beliefs, awareness of inaccurate perceptions, and cognitive correction to erroneous perceptions. Regular homework sessions will also take place, focusing both on behavioural and cognitive goals, and recorded in diaries. This will be discussed in face-to-face sessions and progress reinforced.

Table 1 Components of the cognitive-behavioural therapy arm (10 sessions)

Topic Indicative content

1. Screening and assessment

2. Problem identification and goals

\section{Psychoeducation}

\section{Exposure task}

5 Exposure task and cognitive awareness 6-9 Exposure task and cognitive restructuring

10. Problem solving (optional) and relapse prevention
Main problem is identified and suitability for treatment determined.

Rationale for exposure therapy and cognitive therapy is provided.

A psychosocial history is completed, including past psychiatric history and developmental history.

Measurable problem and goal statements are developed and exposure therapy is introduced.

Client is introduced to a series of activities to differentiate games of skill from games of chance. Independence of events is demonstrated and the concept of illusions of control introduced.

Review of completed cue exposure task. Problem solving and continuing graded exposure task activities

Continuing graded exposure tasks. ABCD model is introduced as well as the concept of automatic thoughts and cognitive distortions

Review of completed cue exposure tasks and continuing graded exposure tasks. Identification and restructuring of unhelpful cognitions through application of the ABCD model

Behavioural experiments may be introduced to test beliefs where appropriate. These will be carefully designed as to not expose the client to increased risk of gambling and will preferably be counsellor led in the first instance (in vivo).

Introduction to a formal problem-solving technique and to the concepts of 'slips' and 'relapse'. Clients are helped to develop a relapse prevention plan through the identification of early warning signs, identification of useful strategies and planning to ask for help in need. 


\section{Text messaging}

Participants allocated to the text-messaging component will receive an average of two text messages per week from the 3-month assessment until the 12-month assessment. Messages were derived from a study investigating the helpfulness and uptake of 99 change strategies used by gamblers to limit or control their gambling. ${ }^{27}$ The most frequent and helpful change strategies for those who were actively attempting to change (classified as action state of change) as well as up to 2 years postrecovery (maintenance) were identified and ranked according to the most helpful and most frequently used. Six change strategies and 56 actions were identified including planning, delay, connecting with others, engaging with alternative activities, cognitive strategies and wellbeing (see table 2 for a full list).

Messages were examined for readability and relevance with a consumer, four counsellors and an experienced team leader. The language of eight items was amended, an item on self-exclusion was deleted (it was assumed to have already been undertaken) and a reminder to recontact services was added to the cognitive condition. Messages were also developed that were tailored to the treatment condition. MI participants will receive messages that encourage self-reflection on treatment goals and workbook use. CBT participants will receive encouragement for using cognitive and behavioural strategies. Messages will be personalised and also start with the wording "some people find this helpful to..." which will take into account that participants may already have implemented the change strategy.

\section{Therapists}

This study was conceptualised as a pragmatic trial to determine the effectiveness of these treatments in reallife settings. For this reason, all experienced therapists from the Oasis treatment services will be trained to deliver MI and CBT treatments. To remove potential contamination of the RCT design by therapist effects, therapists will deliver both treatments. Prior to starting work on the trial, therapists will receive a minimum of 7 days training over three blocks of time. Training will be provided by investigators $\mathrm{MB}(\mathrm{CBT})$ and $\mathrm{DCH}(\mathrm{MI}+\mathrm{W}$ $+\mathrm{B})$ who developed the interventions and are international experts in their field. Ongoing supervision by trained and experienced clinical psychologists will be provided to ensure consistency in the delivery of both interventions. A key advantage of this study is that all therapists offer both interventions. Any bias due to therapist effects (where more or less skilled therapists are associated with one treatment) is minimised. This potential confound will also be reduced by involving multiple counsellors across a number of sites as well as extensive fidelity checks that treatment is being delivered as per protocol. Sessions will be recorded and protocols for assessing treatment fidelity applied and one-third of the fidelity checkers reports being double checked for interrater reliability and fidelity. For $\mathrm{MI}+\mathrm{W}+\mathrm{B}$, recordings will be coded based on the Motivational Interviewing Treatment Integrity (MITI) scale $^{28}$ and for the CBT intervention a competence checklist used in the pilot RCT will be administered. ${ }^{29}$

\section{Baseline assessment}

Baseline measures will be administered by trained research assistants and the data will be directly entered into an in-house built database developed specifically for this RCT. Baseline assessment will involve brief demographics (age, gender, ethnicity, marital status, living arrangements, employment, education, income), the eight-item New Zealand Index of Socioeconomic Deprivation for Individuals, ${ }^{30}$ gambling impacts (ie, work, social life, family and physical health) ${ }^{31}$ and past help seeking. A brief gambling history will be obtained including length of gambling problem, type/s of gambling causing problems, number, nature and outcomes of past attempts to quit or reduce gambling and past treatment and mutual help involvement. Participants will be asked to nominate a goal: stop all forms of gambling, stop only problematic forms of gambling, or reduce their gambling habits. Belief in likelihood of achieving treatment goal, motivation for goal and sense of control over gambling will be measured with a readiness ruler (0 'not at all confident' to 10 'extremely confident'; 'no control' to 'total control'). Primary outcomes of days spent gambling and amount of money spent per day gambling will be measured over the previous 2 months using the timeline follow back procedure. ${ }^{32}$ Pretreatment scores will be calculated, averaged over the 2 months prior to entry. Problem gambling will be determined by the Problem Gambling Severity Index (PGSI), ${ }^{33}$ with categories corresponding to $0=$ no risk; $1-$ 2=low risk; $3-7=$ moderate risk; $8-27=$ problem gambler. The primary outcome for the follow-up study at 12 months will be the dichotomised indicator of problem gambler (PGSI 8-27) versus no risk to moderate risk $(\mathrm{PGSI}<8)$.

Other gambling measures include the Gambling Urge Scale (GUS) ${ }^{34}$ where higher scores indicate greater urges to gamble with a range $0-42$. The GUS has demonstrated concurrent, predictive and criterion validity among non-clinical gamblers and has also been used successfully in clinical samples. ${ }^{35}$ Gambling-related $\operatorname{cog}$ nitions will be measured with the Gambling Related Cognitions Scale (GRCS) which is a 23-item seven-point Likert scale that records the degree of agreement with common thoughts associated with gambling disorder. ${ }^{36}$ The mood and alcohol modules of the Primary Care Evaluation of the Mental Disorders (PRIME-MD) ${ }^{37}$ will be administered to provide diagnoses of major depressive disorder, dysthymia, minor depressive disorder and alcohol abuse/dependence. This is a structured interview designed for primary care clinicians and researchers to diagnose these and other current Diagnostic and Statistical Manual of Mental Disorders (DSM) mental health disorders. It has been validated against the 
Table 2 Text messages for $\mathrm{Ml}$ and CBT groups*

\section{Message content}

Stage 1: CBT arm

specific
Some people find it helpful to complete an automatic thought diary exercise in their exercise book if they notice any gambling related thoughts. Txt STOP to opt out of these messages.

Nip gambling urges in the bud and revisit some of their exposure tasks or the gambling cycle in their exercise book to see if they can identify the trigger.

Remember that if they experience an urge to gamble, it will eventually subside by itself without gambling. Sit with it and allow it to pass.

Limit access to cash when performing new exposure tasks, particularly during early stages of the exposure programme. This will allow you to grade it appropriately.

Remember that the more you put in the more you will get out, so try and do a task from the exercise book every day.

Remember that repeated habituation through performing exposure tasks will eventually lead to conquering gambling urges.

Remember that lapses or 'bumps' are no big deal.

Remember that repeated habituation will eventually lead to extinction. Allowing yourself to experience your urge by focusing on it without gambling is called habituation.

Ask what is the evidence for and against this thought if they notice a thought that tempts them to gamble. Are you jumping to conclusions without looking at all the facts?

Remember the difference between games of skill and games of chance. In games of chance, there is nothing you can do to influence or predict the outcome.

Stage 1: MI arm specific Some people find it helpful to re-read sections of the Becoming a Winner: Defeating Problem Gambling self-help workbook you received. Txt STOP to opt out of these messages.

Read the section on Dealing with Urges in the Becoming a Winner workbook.

Remind themselves periodically about the benefits and costs of gambling described in the Becoming a Winner workbook.

Think again about the ideas provided in Becoming a Winner for limiting access to money. Consider whether there are other life issues that they should address (see Becoming a Winner). Review and revise their plan for dealing with slips that they made in Becoming a Winner. Motivating to share their personal gambling goals with other people (Becoming a Winner). Remind themselves of some of the consequences of their gambling that they listed in Becoming a Winner.

Think about where they would like to be in life in 5 years and whether gambling might interfere with this.

Re-read how they responded to the questions they completed in the Becoming a Winner workbook.

Stage 1: plan and delay Take it easy on yourself or take it slow.

Distract yourself or do something else until the urge to gamble passes.

Postpone gambling until a later date to allow the urge to pass.

Avoid gambling when feeling down, depressed or otherwise vulnerable.

Avoid the first bet because the urge will pass soon enough.

Do things that are incompatible with gambling like meeting a friend, taking a walk without cash or having an early night.

Plan ahead-leave credit cards and non-essential cash at home.

Keep busy to avoid thinking about or engaging in gambling.

Be ready to implement your own personal strategies to deal with gambling triggers.

Count days since you have made a change in your gambling.

Keep track of money by setting up a budget and tracking spending.

Plan ahead and limit the amount of money you carry.

Plan to spend more time with other people.

Stage 2: cognitive
Think about how your money could be better spent.

Remain hopeful about your future.

Remind yourself that you do not need to gamble.

Re-establish trust and belief in yourself.

Make a resolution to continue changing your gambling.

Make a daily affirmation such as staying positive or letting go.

Focus on not gambling each day at a time.

Concentrate on being strong or using will power.

Monitor how your emotions relate to gambling.

Monitor for signs that gambling is becoming a problem again.

Recontact your counsellor or peer support if a check-in could be helpful.

Focus on regaining trust with family and friends.

Continue to accept that you had a problem and move beyond testing yourself. 
Table 2 Continued

Message content

Stage 2: connect and engage

Stage 3: cognitive

Stage 3: well-being
Tell a trusted friend or family member about the extent of your gambling.

Be more open and honest with family and friends about gambling.

Ask family or friends to help or support you.

Engage in an activity that gives you a feeling of achievement.

Participate in regular structured groups or sports like football or tennis.

Engage in a new form of entertainment.

Explore additional work or study-related activities.

Listen, play, sing or dance to music.

Complete daily activities around the house.

Start a new hobby like arts, crafts or knitting.

Plan a reward or something to look forward to.

Taking a long walk can feel good as well as provide a chance to clear your head.

Practise relaxation strategies like yoga, meditation or mindfulness.

Remember that if you are triggered by feeling sad, lonely, angry, hungry or tired, gambling is not the best option.

Monitor your progress. Have you had a change in the urge to gamble?

Calculate your time and money spent gambling: this can really help put it into perspective.

Keep a budget of your spending. This can help you to stay honest.

Acknowledge when you are feeling flat or lonely. This can make you think twice about gambling.

Tell yourself how much better off you will be: healthier, wiser and with more money.

Reflect on differences since changing your gambling: when someone asks to go to the movies, it is nice to be able to say yes and not 'no I am broke'.

Save for a reward like a holiday or a dinner out.

Think about the types of things that you could spend your money on.

Make a goal sheet and then mark off your achievements.

Ensure your physical health needs are met.

Set up and regularly contribute towards a savings plan.

Eat a healthy balanced diet that makes you feel good.

Focus on managing issues that contribute to your gambling.

Learn to forgive yourself for past actions and focus on the future: tomorrow is what you can change, not yesterday.

Be mindful of increasing fluid intake.

Engage in regular exercise.

*Each message started with "some people find this helpful..."

$\mathrm{CBT}$, cognitive-behavioural therapy; MI, motivational interviewing.

Structured Clinical Interview for the DSM-IV. ${ }^{38}$ The use of psychotropic medication and history of manic episodes will be assessed using questions modified from the PRIME-MD $^{37}$ and previously administered in the Gambling Impact Study. ${ }^{39}$ A brief version of the 10 -item Drug Abuse Screening Test (DAST) ${ }^{40}$ will be administered as well as questions about lifetime and current tobacco use and any previous success at quitting problem behaviour (smoking, alcohol, other drugs or other behaviour).

The Kessler 10 (K10) ${ }^{41}$ questionnaire will be included to provide a continuous measure of general psychological distress that is responsive to change over time. Quality of life will be assessed by the EUROHIS-QOL 8-Item Index, ${ }^{42}$ an eight-item version of a widely used measure. Health status will be assessed by the EuroQol EQ-5D-5L, ${ }^{43}$ a five-item measure across the domain of living (ie, mobility, self-care, usual activity, pain/discomfort and anxiety/depression). Self-reported data on direct and indirect costs are associated with productivity losses (eg, days off work) and out-of-pocket expenses such as amount of money spent on gambling and transportation costs.

\section{Follow-up assessments}

Trained research assistants blinded to the client intervention will administer measures at 3 and 12 months. At each follow-up assessment, days gambled and money spent will be assessed with a timeline follow-back interview. Post-treatment scores will be calculated for the month following entry into the trial, in the period from 1 to 3 months and in the interval from 3 to 12 months. Rulers (on a scale of 1-10) will be administered to determine the degree that treatment goals were met (not at all, partially, mostly, completely) and also the sense of control over gambling. Other screens readministered will include PGSI, gambling impacts ${ }^{31}$ GUS, GRCS, PRIME-MD, K10, EUROHIS-QOL 8-Item Index, current tobacco use and cost-effectiveness questions. Participants will also indicate whether they have sought other treatment for gambling, tobacco smoking, alcohol, other drugs or other behaviour. Information on workbook 
engagement will also be collected (whether it had been read, procedures followed, strategies used as well as most and least helpful content) as well as the usefulness of text messaging at 12 months.

After the 3 and 12 months assessments, at least one collateral person per participant (when provided) will be contacted by telephone and asked about the participant's involvement with gambling over the past month. They will also be asked whether he/she gambled noticeably less, at about the same level, or noticeably more than in the past assessment period, and how confident they are about the accuracy of their reports (not at all, somewhat, fairly, extremely). Agreement between collateral and participant reports on days gambled and amount of money spent in the past month will subsequently be assessed using interclass correlation coefficients. Participants who decide to withdraw from the trial, either during the period of intervention delivery or at some point in the subsequent year, will be asked (either by their counsellor or by an AUT research assistant) if they could provide some information as to why they have chosen to withdraw. Any adverse outcomes are likely to be limited to emotional/psychological distress by the participants during the assessment and/or follow-up interviews. In such situations, the researchers will terminate the interview and suggest that participants contact their counsellor at Oasis or the Gambling Helpline, or will offer to contact a service on their behalf to arrange a call back.

\section{Blinding}

Participants will be aware of the trial arm they are assigned to, although neither arm will be described to participants as superior. Researchers blind to participants' treatment groups will conduct baseline and follow-up assessments.

\section{Statistical analysis}

Baseline covariate and outcome values will be tabulated according to treatment groups, along with pooled SDs for continuous outcomes. Missingness and attrition will be reported by treatment group. Unadjusted results per treatment group (CBT vs $\mathrm{MI}+\mathrm{W}+\mathrm{B}$, text messaging vs none, and full $2 \times 2$ factorial breakdown) will be presented with SEs. Should non-normality of residuals be evinced (based on graphical assessment and standard tests), alternative exponential family models with identity link will be preferred to any change in the link function, to avoid effect attenuation associated with averaging over the link, typical of generalised estimating equation (GEE) regression.

The primary analysis set will be the ITT analysis set, comprising all participants, with their original treatment allocation. All planned efficacy analyses will be carried out in the ITT set.

The primary outcomes will be regressed simultaneously in a multivariate model. Measurements at 3 and 12 months will be included in the model to improve statistical efficiency. The primary and secondary hypotheses will be tested and relevant contrasts, including treatment effect differences at 12 months for each outcome, estimated from regression models fitted using GEEs to regress the outcome measurements on the $\mathrm{CBT} / \mathrm{MI}$ intervention factor and the text messaging intervention factor, adjusting for baseline value of the respective outcomes. Primary inference will focus on detecting treatment effects severally in each primary outcome, applying false discovery rate control at the nominal 5\% level. ${ }^{44}$ A secondary inference will consist in producing the observed significance level for the treatment effect on both primary outcomes simultaneously using an F-test or equivalent.

Survival analysis of withdrawal will be applied to identify any prerandomisation and postrandomisation withdrawal predictors. Missing outcome and baseline data will be multiply imputed using prerandomisation and postrandomisation outcome information and withdrawal predictors ${ }^{45}$ treatment assignment, ${ }^{46}$ and demographic and other baseline covariates, under an assumption of missingness at random. ${ }^{47}$ Ten imputed data sets will be generated using a full conditional specification ${ }^{48}$ on these variables, although this number may be revised after a blind review of the data.

Only two subgroups will undergo a planned efficacy analysis: Māori participants and Pacific participants. For this purpose, ethnicity will be prioritised. Subgroup analyses will be carried out using interaction of treatment with subgroup identifiers. The primary purpose of the analysis is to identify the presence of an effect in the subgroup rather than test subgroup heterogeneity.

Mediation analyses will take place on primary and selected secondary outcomes, the latter including problem gambling severity and measures of substance abuse. Mediation analyses will be carried out using multivariate regression analyses involving both direct pathways between intervention arm and outcome, and similar but indirect pathways mediated by participation measures. ${ }^{49}$

\section{Sample size justification}

In accordance with the primary inference, we base the sample size justification on an actual significance level of $2.5 \%$, which will yield a conservative sample size in regard to false discovery rate control. We do not account for the gain in efficiency inherent in modelling data at 3 and 12 months, also a conservative simplification. We will account for observed correlations with baseline values, for which we will make an adjustment of 0.04 in days gambled and 0.49 in money spent. ${ }^{16}$ We plan to recruit a sample of 300 participants and expect to reassess $270(90 \%)$ at 3 months and $225(75 \%)$ at 12 months. These estimates are based on the New Zealand telephone RCT where retention was $88 \%$ at 3 months, $70 \%$ at 12 months and around $50 \%$ at 36 months. The trial sample size (targeted recruitment of 300 yielding 225 complete assessments at 12 months; 
112 per treatment) is sufficient to detect a difference at 12 months between two arms of 1.7 gambling days per month (ES 0.25) and $\$ 7.90$ per day in money spent (ES 0.13 ) with $80 \%$ power in the primary analysis. Assuming participant proportions of $40 \%$ for Māori and $12 \%$ for Pacific people (based on expected percentage of people accessing the Oasis service), and accounting for these two subgroups in terms of testing multiplicity, we will be able to detect a difference of 2.2 days (ES 0.32) and $\$ 12.50$ (ES 0.2) in Māori, and of 3.2 days (ES 0.46) and $\$ 20$ (ES 0.32) in Pacific people with $80 \%$ power.

\section{Data management}

Screening details required by Oasis and the treatment allocation information will be accessed by counsellors from the secure online database hosted at AUT ahead of the client's scheduled appointment. Prior to starting treatment, clients will give additional written consent for their participation in the clinical trial. No identifying personal information will be included in the reporting of information gathered as part of this clinical trial. All participant data will be aggregated prior to being reported. Digital data will be stored only on modern cryptographically strong password-protected and limited access computer systems at the research office. At conclusion of the project, an anonymised version of the data set is available to external parties via the Ministry of Health funding body.

\section{Data monitoring}

An independent Data Monitoring Committee (DMC) will be established by the Steering Committee, composed minimally of a biostatistician, a psychologist and a data manager, none of whom are involved with the study. The DMC Charter will be drawn by the study Steering Committee and define the DMC mandate as consisting of the following duties: monitoring data quality, monitoring attrition, monitoring adverse events and advising the Steering Committee as to trial conduct. There are no formal interim analyses planned, as it is not expected that adverse events will be differentially causally related to the interventions.

\section{DISCUSSION}

To the best of our knowledge, this is the first large-scale trial that will compare the effectiveness of two welldeveloped treatment approaches as well as postcare text messaging. A strength of this study is its delivery in a community treatment setting. Therapists from community agencies will deliver both of the interventions, and through this study have access to training and supervision in these treatments. However, this approach increases the risk that there will be therapy leakage reducing the differentiation between the interventions. To address this issue, we have invested in extensive training and ongoing supervision as well as extensive fidelity checks with the use of validated tools.
This study addresses many of the limitations present in previous gambling treatment research including issues associated with small sample sizes, heterogeneous samples and single site clinical trials. This study does not include a wait-list control because it would not be ethical to include a wait-list control group in a study involving treatment seekers recruited from a community agency. However, the two interventions have been evaluated previously in efficacy trials that included wait-list controls. Working with a large treatment agency is an important strength of this study; however, it does pose some risks in terms of rate of recruitment. In New Zealand, the gambling service system has undergone some changes in the past 2-3 years, including the establishment of a single national telehealth centre that encompasses almost all helpline services for mental health and addiction. Maintaining a flow of clients into the study relies on current numbers of clients being referred to Oasis. It is our preference to maintain the current protocol as we believe recruitment through one large multisite setting strengthens this study, but other recruitment options will be explored if required. Minimising attrition in follow-up assessments is vital to ensure the success of this trial. The at-risk and transient nature of the target population makes this task more difficult. To mitigate this, participants will provide a collateral person who can be contacted.

Despite these limitations, this trial has the potential to make a significant contribution in terms of addressing problem gambling and improving the lives of people impacted by this disorder. It has the potential to provide people with problem gambling access to better treatment. This research also aims to extend current knowledge and inform policy and practice which can lead to more cost-effective outcomes as well as a reduction in gambling harms. Cultural considerations are also of importance given the higher risk among Māori and Pacific people to develop problem gambling.

Date and version identifier: 2.0; 8 June 2015.

\section{Author affiliations}

${ }^{1}$ Gambling and Addictions Research Centre, National Institute for Public Health and Mental Health Research, School of Public Health and Psychosocial Studies, Auckland, New Zealand

${ }^{2}$ Faculty of Health and Environmental Sciences, Auckland University of Technology, Auckland, New Zealand

${ }^{3}$ Department of Biostatistics and Epidemiology, School of Public Health and Psychosocial Studies, Auckland, New Zealand

${ }^{4}$ Health Intelligence and Informatics, Ko Awatea, Counties Manukau District Health Board, Auckland, New Zealand

${ }^{5}$ Department of Psychology, University of Calgary, Calgary, Canada

${ }^{6}$ Department of Psychiatry, Flinders Human Behaviour and Health Research Unit, Flinders University, Adelaide, South Australia, Australia

Contributors MA, DCH and MB developed the idea for this trial and MA sought and obtained funding. MA, MB, DCH, MB and ACV wrote the study protocol. SNR contributed to the text messaging study protocol. The first draft of this article was written by SNR with input from MA and MB. All authors contributed to redrafts of this report.

Funding This work was supported by the Ministry of Health, New Zealand, grant number 352593/00. The Ministry can be reached by emailing Dr Tai Kake at tai_kake@moh.govt.nz. 
Competing interests None declared

Ethics approval Ministry of Health, Health and Disability Ethics Committees.

Provenance and peer review Not commissioned; externally peer reviewed.

Open Access This is an Open Access article distributed in accordance with the Creative Commons Attribution Non Commercial (CC BY-NC 4.0) license, which permits others to distribute, remix, adapt, build upon this work noncommercially, and license their derivative works on different terms, provided the original work is properly cited and the use is non-commercial. See: http:// creativecommons.org/licenses/by-nc/4.0/

\section{REFERENCES}

1. Grinols EL, Smith G, Hodgins D, et al. Social and economic impacts of gambling. In: Smith G, Hodgins DC, Williams RJ, eds. Research and measurement issues in gambling studies. New York: Academic Press, 2007:515-39.

2. Williams RJ, Volberg RA, Stevens RM. The population prevalence of problem gambling: methodological influences, standardized rates, jurisdictional differences, and worldwide trends. Canada: Ontario Problem Gambling Research Centre, 2012.

3. Abbott M, Bellringer M, Garratt N, et al. New Zealand 2012 National Gambling Study: gambling harm and problem gambling: report number 2. Auckland: Auckland University of Technology, Gambling and Addictions Research Centre, 2015.

4. Rossen F. Gambling and problem gambling: results of the 2011/12 New Zealand Health Survey. Auckland, New Zealand: Prepared for the Ministry of Health, Centre for Addiction Research, Auckland UniServices Limited, The University of Auckland, 2015.

5. Kessler RC, Hwang I, LaBrie R, et al. DSM-IV pathological gambling in the National Comorbidity Survey Replication. Psychol Med 2008;38:1351-60.

6. Petry NM, Stinson FS, Grant BF. Comorbidity of DSM-IV pathological gambling and other psychiatric disorders: results from the National Epidemiologic Survey on alcohol and related conditions. J Clin Psychiatry 2005;66:564-574.

7. Problem Gambling Research and Treatment Centre (PGRTC). Guideline for screening, assessment and treatment in problem gambling. Clayton: Monash University, 2011.

8. Cowlishaw S, Merkouris S, Dowling N, et al. Psychological therapies for pathological and problem gambling. Cochrane Database Syst Rev 2012;(11):CD008937.

9. Smith DP, Battersby MW, Harvey PW, et al. Cognitive versus exposure therapy for problem gambling: randomised controlled trial. Behav Res Ther 2015;69:100-10.

10. Ladouceur R, Sylvain C, Boutin C, et al. Group therapy for pathological gamblers: a cognitive approach. Behav Res Ther 2003;41:587-96.

11. Ladouceur R, Lachance S. Overcoming your pathological gambling: workbook. New York: Oxford University Press, 2006.

12. Hodgins DC, Currie S, el-Guebaly N, et al. Brief motivational treatment for problem gambling: a 24-month follow-up. Psychol Addict Behav 2004;18:293-6.

13. Hodgins DC, Currie SR, Currie G, et al. Randomized trial of brief motivational treatments for pathological gamblers: more is not necessarily better. J Consult Clin Psychol 2009;77:950-60.

14. Hodgins DC, Currie SR, El-Guebaly N. Motivational enhancement and self-help treatments for problem gambling. J Consult Clin Psychol 2001;69:50-7.

15. Hodgins DC, Currie SR, el-Guebaly N, et al. Does providing extended relapse prevention bibliotherapy to problem gamblers improve outcome? J Gambl Stud 2006;23:41-54.

16. Abbott M, Bellringer M, Hodgins D, et al. Effectiveness of problem gambling brief telephone interventions: a randomised controlled trial. New Zealand: Prepared for the Ministry of Health, 2012.

17. Petry NM, Weinstock J, Ledgerwood DM, et al. A randomized trial of brief interventions for problem and pathological gamblers. J Consult Clin Psychol 2008;76:318.

18. Toneatto $\mathrm{T}$, Gunaratne $\mathrm{M}$. Does the treatment of cognitive distortions improve clinical outcomes for problem gambling? J Contemp Psychother 2009;39:221-9.

19. Westphal JR, Abbott MW. Models for multi-site gambling clinical trials. Int Gambl Stud 2006;6:129-45.

20. Abbott MW, Clarke D. Prospective gambling research: contribution and potential. Int Gambl Stud 2007;7:123-44.

21. Abbott MW, Williams MM, Volberg RA. A prospective study of problem and regular nonproblem gamblers living in the community. Subst Use Misuse 2004;39:855-84.
22. Agyapong VI, McLoughlin DM, Farren CK. Six-months outcomes of a randomised trial of supportive text messaging for depression and comorbid alcohol use disorder. J Affect Disord 2013;151:100-4.

23. Whittaker R, Dorey E, Bramley D, et al. A theory-based video messaging mobile phone intervention for smoking cessation: randomized controlled trial. J Med Internet Res 2011;13:e10.

24. Whittaker R, Merry S, Stasiak K, et al. MEMO-a mobile phone depression prevention intervention for adolescents: development process and postprogram findings on acceptability from a randomized controlled trial. J Med Internet Res 2012;14:e13.

25. Free C, Knight R, Robertson S, et al. Smoking cessation support delivered via mobile phone text messaging (txt2stop): a single-blind, randomised trial. Lancet 2011;378:49-55.

26. Rodda S, Lubman DI, Dowling N. Effectiveness of e-mental health in the treatment of problem gambling. Melbourne, Australia: Victorian Responsible Gambling Foundation, 2016.

27. Lubman DI, Rodda SN, Hing N, et al. Gambler self-help strategies: a comprehensive assessment of self-help strategies and actions. Melbourne: Gambling Research Australia, 2015

28. Moyers T, Martin T, Manuel J, et al. The Motivational Interviewing Treatment Integrity (MITI) Code: Version 2.0. 2003. http://casaa.unm. edu/download/miti.pdf

29. Battersby M, Smith D, Harvey $\mathrm{P}$, et al. Cognitive versus exposure therapy for problem gambling: a pilot randomised controlled trial. Victoria, Australia: Victorian Responsible Gambling Foundation, 2013.

30. Salmond C, Crampton P, King P, et al. NZiDep: a New Zealand index of socioeconomic deprivation for individuals. Soc Sci Med 2006;62:1474-85

31. Hodgins DC. Reliability and validity of the Sheehan disability scale modified for pathological gambling. BMC Psychiatry 2013;13:177.

32. Sobell LC, Sobell MB. Timeline follow-back. In: Litten R, Allen J, eds. Measuring alcohol consumption. Humana Press, 1992: 41-72.

33. Ferris J, Wynne $\mathrm{H}$. The Canadian problem gambling index. Ottawa, ON: Canadian Centre on Substance Abuse, 2001.

34. Raylu N, Oei TP. The gambling urge scale: development, confirmatory factor validation, and psychometric properties. Psychol Addict Behav 2004;18:100-5.

35. Smith D, Harvey P, Battersby M, et al. Treatment outcomes and predictors of drop out for problem gamblers in South Australia: a cohort study. Aust N Z J Psychiatry 2010;44:911-20.

36. Raylu N, Oei TP. The Gambling Related Cognitions Scale (GRCS): development, confirmatory factor validation and psychometric properties. Addiction 2004;757-69.

37. Spitzer RL, Williams JB, Kroenke $\mathrm{K}$, et al. Utility of a new procedure for diagnosing mental disorders in primary care: the PRIME-MD 1000 study. JAMA 1994;272:1749-56.

38. Spitzer RL, Williams JB, Gibbon M, et al. The Structured Clinical Interview for DSM-III-R (SCID) I: history, rationale, and description. Arch Gen Psychiatry 1992;49:624.

39. Gerstein DR, Hoffmann JP, Larison C. Gambling impact and behavior study: report to the National Gambling Impact Study Commission. National Opinion Research Center, 1999.

40. Skinner HA. The drug abuse screening test. Addict Behav 1982;7:363-71.

41. Kessler RC, Andrews G, Colpe LJ, et al. Short screening scales to monitor population prevalences and trends in non-specific psychological distress. Psychol Med 2002;32:959-76.

42. Schmidt S, Mühlan H, Power M. The EUROHIS-QOL 8-item index: psychometric results of a cross-cultural field study. Eur J Public Health 2006;16:420-8.

43. Rabin $\mathrm{R}$, Oemar $\mathrm{M}$, Oppe $\mathrm{M}$, et al. EQ-5D-5L user guide. Basic information on how to use the $E Q-5 D-5 L$ instrument. Rotterdam: EuroQol Group, 2011: 22.

44. Benjamini $Y$, Hochberg $Y$. Controlling the false discovery rate: a practical and powerful approach to multiple testing. J $R$ Stat Soc $B$ (Methodol) 1995;57:289-300.

45. Carpenter JR, Kenward MG. Missing data in randomised controlled trials-a practical guide. London School of Hygiene, 2007. http:// missingdata.Ishtm.ac.uk/downloads/rm04_jh17_mk.pdf

46. Moons KGM, Donders RART, Stijnen T, et al. Using the outcome for imputation of missing predictor values was preferred. J Clin Epidemiol 2006;59:1092-101.

47. Rubin DB. Inference and missing data. Biometrika 1976;63:581-92.

48. van Buuren S. Multiple imputation of discrete and continuous data by fully conditional specification. Stat Methods Med Res 2007:16:219-42.

49. Lynch KG, Cary M, Gallop R, et al. Causal mediation analyses for randomized trials. Health Serv Outcomes Res Methodol 2008;8:57-76. 